\title{
ENERGY LEVELS AND ELECTRICAL ACTIVITY OF DISLOCATION ELECTRON STATES IN GaAs*
}

\author{
T. Wosiński AND T. FigIELSKI \\ Institute of Physics, Polish Academy of Sciences \\ Al. Lotników 32/46, 02-668 Warszawa, Poland \\ Dedicated to Professor Dr. Julian Auleytner \\ on the occasion of his 70 th birthday
}

(Received August 3, 1992)

\begin{abstract}
Experimental results are presented confirming that the two energy levels in GaAs: $E_{\mathrm{c}}-0.68 \mathrm{eV}$ and $E_{\mathrm{v}}+0.37 \mathrm{eV}$, discovered in plastically deformed crystals, belong actually to dislocations. In view of recent identification of the electron state of misfit dislocations at an InGaAs/GaAs interface, a correspondence between these levels and dislocation types has been reinterpreted. The first mentioned level belongs likely to $\alpha$ while the second one to $\beta$ dislocations of $60^{\circ}$ (glide set) type. Such a correspondence is compatible with the observed effect of irradiation on dislocation glide motion in GaAs. It is also argued that these energy levels are involved in the phenomenon of unquenchability of the EL2 defects placed in high-stress regions near dislocations.
\end{abstract}

PACS numbers: 61.70.-r, 71.55.Eq

\section{Introduction}

Identification of bound electron states induced by dislocation cores in semiconductor crystals is an old-standing problem in solid state physics. Unfortunately, any reliable results concerning this problem are rather scarce even in the case of elemental semiconductors. Usually, during plastic deformation of a crystal a large number of point defects is generated whose electrical activity hides that of the core states of dislocations.

At this moment one might wonder whether interest in the core states of dislocations has any practical importance as those states almost always represent a hidden minority. Of course, people dealing with dislocations should not have the slightest doubt about it. A dislocation represents a dynamic defect which has a property of selfreproducing and is strongly interacting with all other kinds of

"Presented as invited paper at the European Research Conference on "Physical Metallurgy: Extended Defects in Semiconductors", August 1992, Holzhau, Germany. 
defects in a crystal. On the other hand, it has been well established that under certain conditions dislocation dynamics is very sensitive to the concentration of electrons and holes occupying the corresponding energy bands of a semiconductor, which is surely a manifestation of energy levels in the bandgap associated with dislocation cores. Therefore those energy levels can have primary impact on the dislocation dynamics, and by the same on the resulting defect structure of a whole crystal or device.

Two other important cases are also known when the electron states of dislocations could manifest themselves explicitly. One is the process of dislocation-related degradation of optoelectronic devices. The other occurs when misfit dislocations are formed at an interface of a lattice-mismatched heterostructure. In the latter case a charge accumulated at the misfit dislocations could essentially disturb the potential profile across the interface.

In view of what has been said at the beginning, the case of GaAs looks somewhat exceptionally. During a few recent years some reliable information regarding this material has been gleaned which enabled us to try to discuss its certain properties in terms of energy levels of dislocation cores.

\section{Energy levels of dislocations in GaAs}

Probably the first direct observation of the dislocation-related level in the bandgap of GaAs was the one done by Wosinski [1] with the DLTS (deep level transient spectroscopy) technique. We omit here a discussion of earlier results obtained with the Hall effect measurements [2] as weighted down with a greater uncertainty. Wosinsski observed in plastically deformed (at $400^{\circ} \mathrm{C}$ ) single crystals of $n$-type GaAs a hump on the low-temperature slope of the dominant electron-emission peak of the EL2 trap. It was possible to deconvolute the as-observed DLTS signal into two peaks: one characteristic of EL2 and the other, called ED1, induced by the plastic deformation (Fig. 1).

Specific properties of the DLTS signal corresponding to this ED1 electron trap made it possible to relate ED1 to introduced dislocations. The principal feature of the signal was a linear dependence of its amplitude on the logarithm of the filling time of the traps with electrons, observed over six decades (Fig. 2). This nonstandard behaviour points out that ED1 traps are arranged in such a way that the Coulomb interaction between electrons captured at the traps limits their population, which is characteristic of dislocations. Also the number of ED1 traps estimated from the DLTS signal was in reasonable agreement with the number of possible core states along dislocations in the crystal. The DLTS peak of ED1 is essentially broadened which might point out that we have, in fact, to do with one-dimensional energy band.

Later on, another deformation-induced DLTS-peak, called HD1, was revealed by Wosiński in p-type GaAs [3] (Fig. 3). This hole trap displayed again similar features of the DLTS signal as ED1 did, which enabled the author to relate it to dislocations as well.

Let us come back to ED1. Mera et al. [4] observed traps corresponding to ED1 in plastically deformed GaAs crystals but they entertained doubts whether 


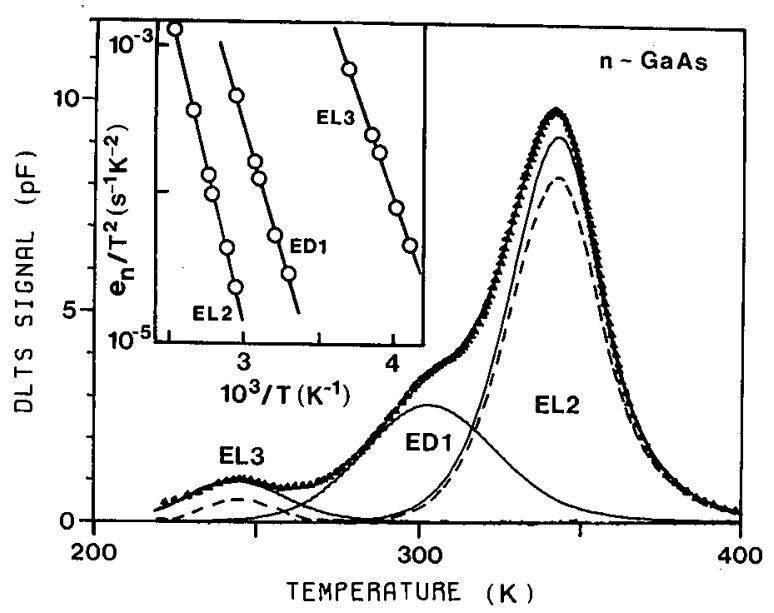

Fig. 1. DLTS spectra of undeformed (dashed line) and plastically deformed to the strain $3.4 \%$ (triangles) $n$-type samples, recorded at the rate window $2.5 \mathrm{~s}^{-1}$. Deconvoluted peaks of the latter spectrum are drawn with solid lines. Thermal activation plots of the electron emission rates are shown in the inset.

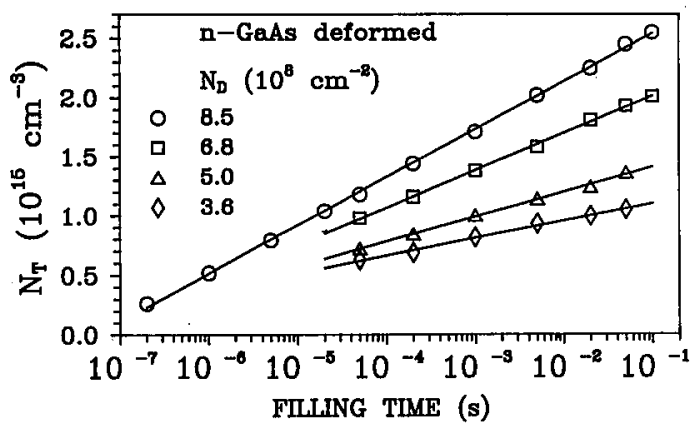

Fig. 2. Concentrations of electrons trapped at ED1 traps vs. filling-pulse duration time measured in various plastically deformed $n$-type samples. Dislocation densities are written in the inset.

these traps actually were associated with dislocations. A serious drawback in a detailed examination of ED1 was that its emission peak coincided badly with that of EL2. Therefore it was absolute necessity to look for ED1 in a material possibly free of EL2. A few attempts to do this have been undertaken by now.

Fang [5] investigated specially prepared Bridgman-grown GaAs crystals with suppressed concentration of EL2. After plastic deformation she observed the DLTS signal corresponding to ED1, whose amplitude essentially exceeded that of EL2.

Fronc et al. [6] identified the ED1 and HD1 traps in a crystal grown by rarely applied SSD (Synthesis, Solute Diffusion) method. This method may be viewed as a modification of the liquid phase epitaxy technique adapted to grow bulk crystals. 


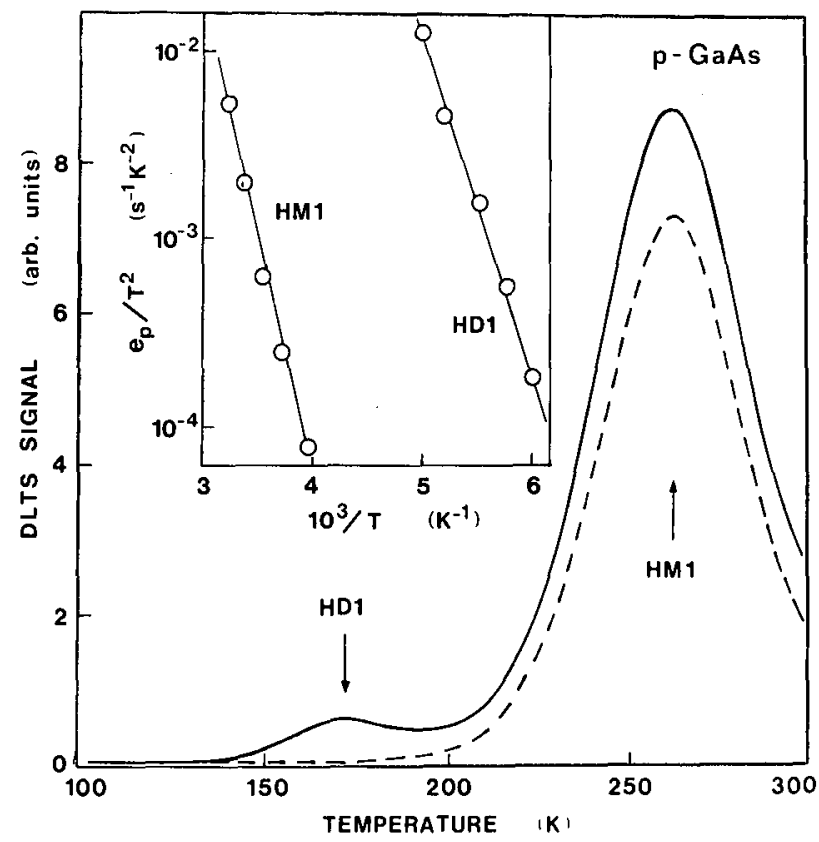

Fig. 3. DLTS spectra of undeformed (dashed line) and plastically deformed to the strain $2 \%$ (solid line) $p$-type samples, recorded at the rate window $17 \mathrm{~s}^{-1}$. Thermal activation plots of the hole emission rates are shown in the inset.

The investigated crystal was completely free of EL2 but contained an enhanced density of grown-in dislocations.

Finally, we have been looking for an electrical activity of dislocations introduced by micro-indentation in thick $(\simeq 1 \mathrm{~mm}) n$-type GaAs layers grown by liquid phase electroepitaxy (LPEE). In the as-grown samples DLTS measurements revealed no deep traps in the upper half of the energy gap with a concentration exceeding the detection limit of $10^{12} \mathrm{~cm}^{-3}$ (Fig. 4). Indentations were performed as in the microhardness test, using a Vickers diamond indenter and $0.5 \mathrm{~N}$ load. An array of $10 \times 10$ indents separated from each other by a distance of $100 \mu \mathrm{m}$ was rinade on the (001) surface of the sample at a temperature of $250^{\circ} \mathrm{C}$ in air. A gold disc was evaporated onto the indented area to form Schottky diode. DITS measurements performed with this diode revealed just the ED1 trap at a concentration of about $10^{13} \mathrm{~cm}^{-3}$ as a dominant deep trap introduced by the indentation (Fig. 4).

The up-to-date parameters of the both dislocation-related traps in GaAs are presented in Table. After accepting the dislocation origin of these two traps, a next question arises as to which particular type of dislocations the traps belong. In the 


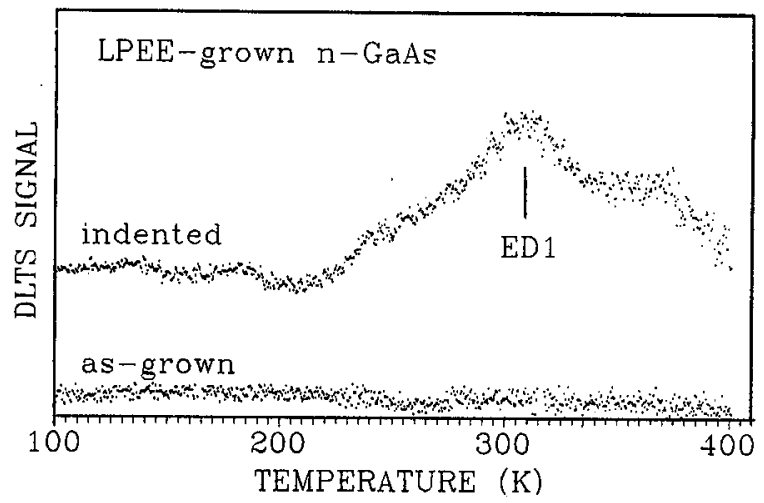

Fig. 4. DLTS spectra of LPEE-grown $n$-type GaAs recorded at the rate window $11 \mathrm{~s}^{-\mathbf{1}}$. Lower spectrum corresponds to as-grown sample and the upper one to the sample subjected to local plastic deformation by micro-indentation.

TABLE

DLTS parameters of dislocation-related traps in GaAs.

\begin{tabular}{c|c|c|c}
\hline \hline $\begin{array}{c}\text { Trap } \\
\text { name }\end{array}$ & $\begin{array}{c}\text { Apparent energy } \\
\text { level [eV] }\end{array}$ & $\begin{array}{c}\text { Capture cross } \\
\text { section }\left[\mathrm{cm}^{2}\right]\end{array}$ & $\begin{array}{c}\text { Full peak half } \\
\text { width [meV] }\end{array}$ \\
\hline ED1 & $E_{\mathrm{c}}-0.68( \pm 0.01)$ & $2 \times 10^{-14}\left( \pm 1 \times 10^{-14}\right)$ & $80( \pm 10)$ \\
IID1 & $E_{\mathrm{v}}+0.37( \pm 0.01)$ & $2 \times 10^{-14}\left( \pm 1 \times 10^{-14}\right)$ & $100( \pm 20)$
\end{tabular}

samples investigated by now there was always a domination of $60^{\circ}$ dislocations, thus - if to ignore a complication resulting from their possible dissociation into partials - we have a choice between $\alpha$ and $\beta$ dislocations. Those dislocation types correspond respectively to $\mathrm{As}$ and $\mathrm{Ga}$ atoms at the edge of an extra lattice plane of $60^{\circ}$ dislocations, assuming that they belong to the dominating glide set.

In the first papers $[1,3]$ the ED1 trap was tentatively ascribed to $\beta$ dislocation and the IID1 trap to $\alpha$ dislocation. The argumentation for such a correspondence was based on the fact that an average velocity of grown-in dislocations in GaAs crystals depended on the doping level but differently for $\alpha$ 's and $\beta$ 's [7]. This effect has been commonly assumed to be associated with a Fermi level shift inside the bandgap and not with locking the dislocations with impurities. But only the $\beta$ dislocations are those whose velocity changes by several orders of magnitude as the Fermi level sweeps over the upper half of the bandgap. Thus, it was very natural to suppose that energy level of $\beta$ 's lies higher than that of $\alpha$ 's, and such a sequence was indeed predicted by theoreticians [8]. However, these arguments seem to be refuted in light of recent experimental results discussed in the following. 


\section{Reinterpretation of the former results}

Watson et al. [9] observed deep level states induced undoubtedly by misfit dislocations formed between InGaAs and GaAs layers grown by MOCVD technique. They could change the density of $\alpha$ and $\beta$ dislocations separately by altering the size of rectangular mesas etched from the substrate. Using the DLTS spectroscopy they found an electron trap with an energy level at $0.58 \mathrm{eV}$ below the conduction band edge, whose concentration correlated with the density of $\alpha$ dislocations and which was being filled in the manner characteristic of dislocations. Although this level is $0.1 \mathrm{eV}$ shallower than the ED1 level in GaAs, they both seem to belong to a trap of the same nature. The difference in their activation energies may be naturally connected with a narrower (by $0.095 \mathrm{eV}$ ) energy gap of the ternary compound of the epitaxial layer.

Those results confirm further the dislocation origin of the ED1 trap in GaAs, but they require a reinterpretation of the originally proposed correspondence of the ED1 and HD1 traps with the particular dislocation types. In fact, the cited results demonstrated with a great confidence that the dislocation-dependent deep level observed there (analogue of ED1), was associated with $\alpha$ dislocations! Thus our higher level, ED1, would also correspond to $\alpha$ dislocations, while the lower one, HD1, probably to $\beta$ dislocations. We shall show in the following that such an "inverted" correspondence is compatible with another phenomenon involving the motion of dislocations in GaAs, being a kind of the photoplastic effect.

In $\mathrm{GaAs}$ this effect was thoroughly investigated by Maeda et al. [10, 11]. In those experiments single crystals of GaAs were subjected during the deformation to irradiation with electron beam or intense light generating electron-hole pairs. The authors were looking for radiation-induced changes in the dislocation velocities under applied stress at variable temperature. The principal results of those investigations are following. In a low temperature range the activation energy of dislocation motion is substantially reduced by irradiation. This reduction depends on the type of dislocation involved: $\alpha, \beta$ or screw, but it is independent of the irradiation intensity. Only preexponential factor of this thermally activated process rises almost proportionally with the irradiation intensity.

Before we shall discuss the results of those experiments, let us remind what is the dominating mechanism of dislocation glide in GaAs. It is rather commonly accepted that in a low temperature range the dislocation glide is actuated by thermal formation of kink pairs and subsequent kink migration along the dislocation lines. Both these elementary steps of dislocation motion involve bond switching, and are thermally activated.

The studies of photoplastic effect by Maeda et al. $[10,11]$ point out to a recombination enhanced mechanism as its origin. It may be viewed in the following way. The energy released in the electron-hole recombination event at a dislocation is exploited to assist to overcome the potential barrier for a kink formation or migration [12]. Thus this energy should be subtracted from the thermal activation energy necessary for the same process but occurring without irradiation. The higher intensity of irradiation the more frequently those events occur.

We invoke now the quantitative results of those experiments. The authors 
obtained the following values of the energy reduction caused by irradiation: $0.7 \mathrm{eV}$ for $\alpha$ dislocations, and $1.1 \mathrm{eV}$ for $\beta$ dislocations. These values coincide surprisingly well with the energies which are to be released in a nonradiative recombination event occurring through the ED1 and HD1 trap level, respectively!

Thus this result might be considered as an explicit manifestation of the energy levels of dislocations in $\mathrm{GaAs}$, on the understanding that their sequence in the bandgap is inverted compared with that postulated formerly $[1,3]$. Taking into account the finding of Watson et al. [9] and the present argumentation, we arrive at the following correspondence: $\mathrm{ED} 1 \Leftrightarrow \alpha$ dislocations, $\mathrm{HD} 1 \Leftrightarrow \beta$ dislocations.

\section{Dislocation electron states and unquechability of EL2}

To complete this discussion we want to pay attention to one more effect in which the energy levels of dislocations in GaAs play probably an important role. That is the unquenchability of the EL2 defects when placed in a close vicinity of dislocation lines. The famous EL2 trap in GaAs displays an intriguing property that it can be optically transformed into a metastable configuration which afterwards is not electrically or optically accessible. This property is called the EL2 quenching and it has been usually considered as a fingerprint of EL2.

However, there are EL2-like defects generated by plastic deformation, or simply placed near dislocations, which are not quenchable. The question is whether these defects are actually regular EL2 that became unquenchable for some particular reasons, or are they quite different species? In our opinion the first possibility is the case [13], and in the following we present arguments in favour of that.

An acceptor level associated with the metastable configuration of EL2 has been recently discovered by Baj et al. [14]. This level is normally degenerate with the conduction band of GaAs but under a hydrostatic pressure exceeding $0.2 \mathrm{GPa}$ it enters the bandgap. When negatively charged with an electron which occupies this level, the metastable configuration needs much less activation energy $(75 \mathrm{meV}$ instead of $0.35 \mathrm{eV}$ in the neutral state) for the regeneration of its normal (ground) state.

We believe that this is also the case of EL2 placed in a close vicinity of dislocation. In such a case EL2 is subjected to a high quasi-hydrostatic pressure originating from the dislocation. There is considerably large volume of a crystal along each dislocation where the acceptor level of metastable EL2 enters the bandgap. We suppose that such EL2 is then hardly quenchable because of a competition of the regeneration process occurring via the negatively charged metastable state.

An assistance of the dislocation energy levels is then necessary. First, these levels assure that the illumination which transforms EL2 from its normal to metastable state $(h \nu \approx 1.1 \mathrm{eV})$ generates also at the same time electrons (and holes) which may next occupy the acceptor level in question. Thus the activation energy for the regeneration process is strongly reduced. Moreover, the energy released in the recombination of an electron-hole pair through the dislocation level can supply the nearby EL2 defect with a lacking energy if needed. 


\section{Final remarks}

In conclusion, we want to emphasize that GaAs seems to be a rather unique semiconductor in the context considered in this paper. During the plastic deformation of GaAs no other defects than dislocations are produced in a considerable amount having energy levels within the range accessible by the DLTS spectroscopy. This advantage made it possible to identify the energy levels of dislocation electron states, as well as to point out some important phenomena in which these levels are involved. If the presently proposed correspondence of the energy levels with particular dislocation types is correct, then the observed dependence of the dislocation velocity on the doping level requires a new explanation which does not invoke a variable occupation of these energy levels.

\section{Acknowledgements}

The authors would like to thank Dr. Z.R. Żytkiewicz for growing the LPEE layers and Dr. A. Mąkosa for cooperation in the DLTS measurements.

\section{References}

[1] T. Wosiński, J. Appl. Phys. 65, 1566 (1989).

[2] D. Gerthsen, Phys. Status Solidi A 97, 527 (1986).

[3] T. Wosiński, in: Defect Control in Semiconductors, Ed. K. Sumino, North-Holland, Amsterdam 1990, p. 1465.

[4] Y. Mera, K. Miyahara, H. Yasuda, K. Maeda, in: Defect Control in Semiconductors, Ed. K. Sumino, North-Holland, Amsterdam 1990, p. 1471.

[5] L. Fang, Ph.D. Thesis, Göttingen University, Göttingen 1991.

[6] K. Fronc, A. Makosa, T. Wosiński, Acta Phys. Pol. A 80, 349 (1991).

[7] K. Maeda, in: Dislocations in Solids, Ed. H. Suzuki, University of Tokyo Press, Tokyo 1985, p. 425.

[8] R. Jones, A. Öberg, S. Marklund, Philos. Mag. B 43, 839 (1981).

[9] G.P. Watson, D.G. Ast, T.J. Anderson, B. Pathangey, Y. Hayakawa, J. Appl. Phys. 71, 3399 (1992).

[10] K. Maeda, M. Sato, A. Kubo, S. Takeuchi, J. Appl. Phys. 54, 161 (1983).

[11] K. Maeda, S. Takeuchi, J. Physique 44, C4-375 (1983).

[12] N. Maeda, S. Takeuchi, in: Defect Control in Semiconductors, Ed. K. Sumino, North-Holland, Amsterdam 1990, p. 1397.

[13] T. Figielski, T. Wosiński, A. Mąkosa, Phys. Status Solidi A 131, 369 (1992).

[14] M. Baj, P. Dreszer, A. Babiński, Phys. Rev. B 43, 2070 (1991). 